Interfaces

\title{
La photographie : medium d'une exploration du logement, espaces de syntaxes domestiques provisoires
}

Danièle Méaux

\section{CpenEdition}

\section{Journals}

Édition électronique

URL : http://journals.openedition.org/interfaces/1607

DOI : 10.4000/interfaces. 1607

ISSN : 2647-6754

Éditeur :

Université de Bourgogne, Université de Paris, College of the Holy Cross

\section{Édition imprimée}

Date de publication : 15 décembre 2020

Pagination : 79-96

ISSN : $1164-6225$

Référence électronique

Danièle Méaux, « La photographie : medium d'une exploration du logement, espaces de syntaxes domestiques provisoires », Interfaces [En ligne], 44 | 2020, mis en ligne le 15 décembre 2020, consulté le 21 décembre 2020. URL : http://journals.openedition.org/interfaces/1607 ; DOI : https://doi.org/ 10.4000/interfaces.1607

\section{(c)}

Les contenus de la revue Interfaces sont mis à disposition selon les termes de la Licence Creative Commons Attribution 4.0 International. 


\title{
LA PHOTOGRAPHIE : \\ MEDIUM D'UNE EXPLORATION DU LOGEMENT, ESPACES DE SYNTAXES DOMESTIQUES PROVISOIRES
}

\author{
Danièle Méaux \\ Université Jean Monnet, CIEREC
}

\begin{abstract}
Résumé: Le logement est le lieu par excellence d'une appropriation domestique de l'espace s'accompagnant d'agencements précaires, liés à l'existence quotidienne et à des idiosyncrasies personnelles. Des photographes ont choisi d'enquêter sur ces environnements instables et familiers. C'est en particulier le cas de François Hers (Intérieurs, 1981), Jean-Marc Tingaud (Intérieurs, 1991) ou Hortense Soichet (Intérieurs. Logements à la Goutte d'Or, 2011). Le premier réalise des vues en plan moyen aux tonalités criardes. Le second recourt au plan rapproché pour retenir des agencements précaires d'envergure limitée. Enfin, la dernière combine les vues des appartements à des paroles d'habitants qui livrent les bribes de récits de vie. Les procédures employées par ces photographes s'avèrent capables d'engager une réflexion approfondie sur les agencements vernaculaires de ces espaces intérieurs où les objets, le plus souvent fabriqués en série et peu onéreux, s'organisent en une syntaxe individuelle et changeante, liée aux «arts de faire» de leurs habitants.
\end{abstract}

Mots-clefs : photographie, art, vernaculaire, sociologie, espace, habiter, quotidien, enquête, couleur, intérieur

Abstract: The home is the ideal place for a domestic appropriation of space accompanied by delicately arranged layouts linked to daily existence and personal idiosyncrasies. Photographers have chosen to investigate these unstable and familiar environments. This is especially the case for François Hers (Intérieurs, 1981), JeanMarc Tingaud (Intérieurs, 1991) or Hortense Soichet (Intérieurs. Logements à la Goutte d'Or, 2011). The first photographer takes middle distance shots with garish tones. The second uses close-ups to capture small-scale frail arrangements. The third associates shots of apartments with words from the inhabitants giving snippets of their life stories. The processes used by these photographers succeed in provoking a profound reflection on the vernacular arrangements of these interior spaces, where often inexpensive mass-produced objects are organised in an individual and changing syntax, linked to the "arts of making" of their inhabitants.

Keywords: photography, art, vernacular, sociology, space, dwelling, everyday life, inquiry, color, interior 
Pour Jean-Marc Besse, «[1] es humains [...] existent en habitant l'espace, tout l'espace, du plus proche au plus lointain, en le sillonnant de toutes parts, en le transformant, en l'orientant et en l'organisant, en s'y installant de diverses manières [...]» (7). C'est à différentes échelles que l'homme - cet être spatial (Lussault) - investit le monde, mais la sphère du logement possède pour chaque individu une importance singulière: elle constitue un abri, une sorte de bulle protectrice (Moles, Psychologie de l'espace). Pour ceux qui y vivent (même temporairement), elle compose une zone de stabilité, à partir de laquelle il leur est possible d'exister (au sens étymologique de ek-sistere: «se tenir à partir de»). Le logement est également le réceptacle d'objets avec lesquels l'habitant entretient des rapports physiques et quotidiens. Il est l'espace d'une syntaxe privée qui s'est façonnée à partir d'acquisitions diverses, opérées au gré des opportunités ou des besoins éprouvés.

Cette aire privée est souvent partagée par plusieurs personnes; elle résulte alors d'ajustements et de négociations implicites permettant que les meilleurs agencements soient trouvés. Cumulatif et sédimentaire, l'espace du logement comprend des objets utiles, comme des éléments destinés à la décoration ou au souvenir. L'intérieur se présente donc comme un «monde» élaboré à partir d'habitudes quotidiennes et riche de dépôts liés aux itinéraires biographiques de ses occupants. Loin de se résumer à une disposition architecturale, il est matérialisation de modes d'existence et d'appartenances culturelles. Intimement lié à des manières d'être au monde (Illich 66), il est l'espace privilégié d'arrangements à destination personnelle et fonctionnelle, parfois élaborés avec des moyens restreints - souvent qualifiés par le vocable « vernaculaire».

Dans les quarante dernières années se sont développées dans le champ de l'art des pratiques photographiques constituant de véritables enquêtes, problématisées et établies dans la durée (Méaux). Ces travaux manifestent le regain d'intérêt des artistes pour le réel (Foster) et le croisement de leurs préoccupations avec celles des chercheurs en sciences humaines et sociales: anthropologues, sociologues, géographes notamment (Caillet). Il s'agira ici d'examiner les façons dont la photographie peut contribuer à l'exploration des manières d'habiter et d'aménager l'espace domestique. À cette fin, trois séries seront mises en perspective: la première réalisée par François Hers en 1981, la seconde par Jean-Marc Tingaud en 1991 et la dernière par Hortense Soichet en 2011. Si ces trois œuvres entrent en résonnance du fait d'un titre commun - Intérieurs -, leur proximité ne s'arrête pas là : montrant des logements vides de leurs habitants, François Hers, Jean-Marc Tingaud et Hortense Soichet proposent tous trois une investigation minutieuse et acérée de la manière dont ces espaces sont organisés. L'absence même des occupants amène en effet l'observateur à ressentir avec force combien les lieux représentés ne se présentent pas comme des étendues uniformes et homogènes, mais se trouvent orientés à partir de personnes vivantes dont le défaut fait précisément ressortir qu'ils sont à la source de l'organisation des espaces domestiques figurés. L'attention se porte dès lors sur la singularité de chaque aménagement, lié à des manières d'habiter. À 
chaque fois, l'organisation des photographies en série incline le spectateur à l'exercice de la comparaison et au prélèvement des indices signifiants. Ces travaux ont été publiés et l'espace du livre se montre propice au décryptage des photographies et à l'articulation des observations effectuées à partir de chacune d'entre elles.

Échelonnées sur une quarantaine d'années, les séries de François Hers, Jean-Marc Tingaud et Hortense Soichet amènent à balayer une évolution des modes et des goûts en matière d'ameublement, à prendre en compte des disparités découlant de différences de milieux, comme d'aires géographiques et culturelles. La confrontation de ces trois séries renvoie aussi à une histoire de la photographie en couleur. Mais, surtout, les points de vue adoptés sur les logements et les agencements domestiques diffèrent nettement, de sorte que ces trois séries travaillent à penser de façon dialogique ces syntaxes privées, et à appréhender le «vernaculaire» avec nuance. Loin d'illustrer un concept établi a priori, ces trois photographes travaillent à le comprendre et à le définir, dans sa complexité et ses contradictions.

\section{Intérieurs, 1981}

L'ouvrage que le photographe François Hers réalise en collaboration avec Sophie Ristelhueber découle d'une commande des services officiels du logement social dans la partie wallonne de la Belgique, qui était en premier lieu destinée à des chercheurs en sociologie. S'écartant du cahier des charges initialement imparti qui se focalisait sur l'architecture (Bonhomme 91), François Hers se concentre sur l'intérieur des logements. Il en visite un grand nombre qu'il photographie en couleur, de façon systématique, en employant toujours la même optique et un éclairage au flash direct «pour que tous les éléments aient la même valeur et qu'aucune ambiance particulière ne soit privilégiée» (Hers Intérieurs, troisième de couverture); les volumes se trouvent ainsi écrasés au sein des vues. François Hers invite également Sophie Ristelhueber à participer à l'entreprise en s'attachant aux relations des personnes avec leurs espaces de vie. La photographe - qui n'en est alors qu'à ses débuts - commence par rencontrer les habitants afin de se familiariser avec eux; puis elle réalise des vues en noir et blanc et en plan moyen, où les personnes prennent la pose dans leur intérieur. Ses images témoignent du désarroi de visages fatigués et de corps abîmés au sein de leur cadre de vie. La présence des habitants dans les photographies en noir et blanc fait ressortir leur absence dans les vues de François Hers. À la fin du livre, figure également un texte du sociologue René Schoonbrodt qui alimente l'interprétation de la première série.

Équilibrant la part du document, du médium et de l'auteur, François Hers note:

Nous nous sommes sentis libres de nos choix dans ce qui nous avait été demandé: rendre compte d'une réalité sociale et des programmes de construction qui y sont liés. 
Mais ces choix, nous les avons faits en fonction de la nature même de la photographie. Si celle-ci informe - puisque l'on peut toujours reconnaître ce qu'elle imprime avec réalisme - elle est avant tout une affirmation, une émotion qui existe. (Troisième de couverture)

Artiste conceptuel avant de devenir photographe, François Hers a participé à la création en 1972 de l'agence VIVA dont les membres souhaitaient tourner le dos aux normes du photojournalisme pour traiter en profondeur des sujets concernant la vie quotidienne dans ses dimensions politiques ${ }^{1}$. François Hers a été l'instigateur du reportage collectif «Familles de France» (1973) qui reflétait les bouleversements de la société française ; il a aussi participé à l'enquête réalisée sur «Les Français en vacances $»^{2}$ (1976). Pour François Hers, l'art et la photographie se doivent de répondre à une demande sociale. Ces convictions, il les réaffirmera à maintes reprises au fil de son itinéraire: au travers de la Mission photographique de la DATAR (1983-1989), puis de l'aventure des Nouveaux Commanditaires ${ }^{3}$ au sein de la Fondation de France, à partir de 1991.

Dans Intérieurs, les photographies proposées par François Hers sont des plans moyens (relativement serrés cependant) aux couleurs denses voire criardes, effectués sous une lumière homogène et brutale. Ses vues retiennent les tonalités crues des rideaux, des abat-jours, des nappes imprimées, de posters à l'évidente banalité. Si les motifs floraux des papiers peints et des tissus viennent s'entrechoquer dans le champ, d'une vue à l'autre frappe la conformité d'un ameublement qui révèle tout à la fois une absence de réelle pauvreté et l'assujettissement à la consommation d'une gamme de produits standardisés, fabriqués en série au moindre coût. Les objets et les éléments de mobilier s'avèrent très kitsch (Adorno); ils renvoient aussi aux goûts d'une époque. L'ensemble signe une volonté d'approcher l'endotique, le quotidien dont Henri Lefebvre contribua à démontrer l'importance avec Critique de la vie quotidienne dès 1947. Les gestes de tous les jours qui façonnent les consciences (Lefebvre 157) s'accrochent en effet à des objets et à des espaces domestiques.

Alain Dagbert, Martine Franck, Hervé Cloaguen, Claude Raymond-Dityvon, Richard Kalvar, Jean Lattès et Guy Le Querrec travaillent ensemble au sein de cette agence jusqu'en 1978.

2. Ces bourses sont accordées par la Fondation nationale de la photographie à l'occasion du quarantième anniversaire des congés payés.

3. URL: http://www.nouveauxcommanditaires.eu/fr/home/accueil (page consultée le 30 octobre 2020). 


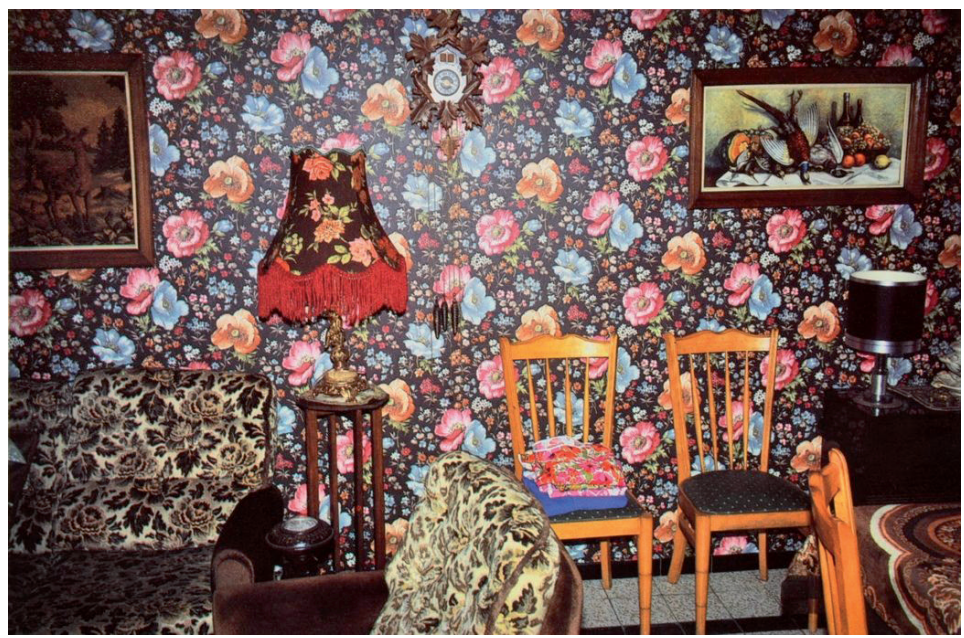

Figure 1. Intérieurs (1981 n.p.).

(C) François Hers.

La frontalité des images donne le sentiment d'une relative absence de sophistication. François Hers confie son intérêt pour l'œuvre du photographe américain Weegee (Hers Intérieurs, 13). Il a également étudié les méthodes d'identification mises au point par Alphonse Bertillon pour la photographie judiciaire à la fin du $\mathrm{XIX}^{\mathrm{e}}$ siècle. Il apprécie en effet le caractère direct et antiartistique de vues faites pour la presse ou pour la police, qui se présentent comme de simples constats; il se montre aussi attiré par la sérialité à même de permettre la constitution d'une archive exploitable. François Hers développe d'ailleurs un parallèle entre l'enquête telle qu'elle est menée en milieu policier et la pratique du reportage : il s'agit pour lui d'un «même goût pour la recherche, l'enquête, le désir de comprendre ce qui se passe vraiment. Sans bien savoir ce qui vous pousse et où cela peut aboutir. Que fait la police ? Elle attend. Les photographes aussi» (Hers Intérieurs 13). Il confie ailleurs : «J'ai pratiqué les techniques de base du travail de la police: regarder sans en avoir l'air, garder son sang-froid, quand l'on vous regarde à votre tour, analyser un comportement, attendre et interroger la réalité» (Hers 1982, 31).

Dans des ouvrages récents, Dominique Kalifa et Luc Boltanski ont rapproché la démarche du sociologue de celle du détective (Kalifa; Boltanski). Le contexte même de la commande comme la présence du texte de René Schoonbrodt signent la proximité du travail photographique et de la socio- 
logie. Le chercheur retrace succinctement les modalités de l'expansion de l'habitat social en réponse aux besoins en logement générés par l'industrialisation galopante du XIX ${ }^{\mathrm{e}}$ siècle. Il pèse les apports et les limites des options urbanistiques retenues, à la périphérie des villes, quand il aurait mieux valu selon lui placer les travailleurs «au cœur même de l'espace politique» (Schoonbrodt 122). Il souligne aussi l'utilité, pour le sociologue, des photographies de François Hers et Sophie Ristelhueber, qui «livrent des renseignements qui échappent aux capacités habituelles des techniques d'enquête». C'est ainsi que leurs travaux «appuient les recherches en sociologie du quotidien en leur donnant une clarté quelque peu effrayante non par la révélation de la persistance de la pauvreté, mais par celle tout aussi angoissante, de l'insertion des habitants des logements sociaux à une place précise de la structure sociale et culturelle.»

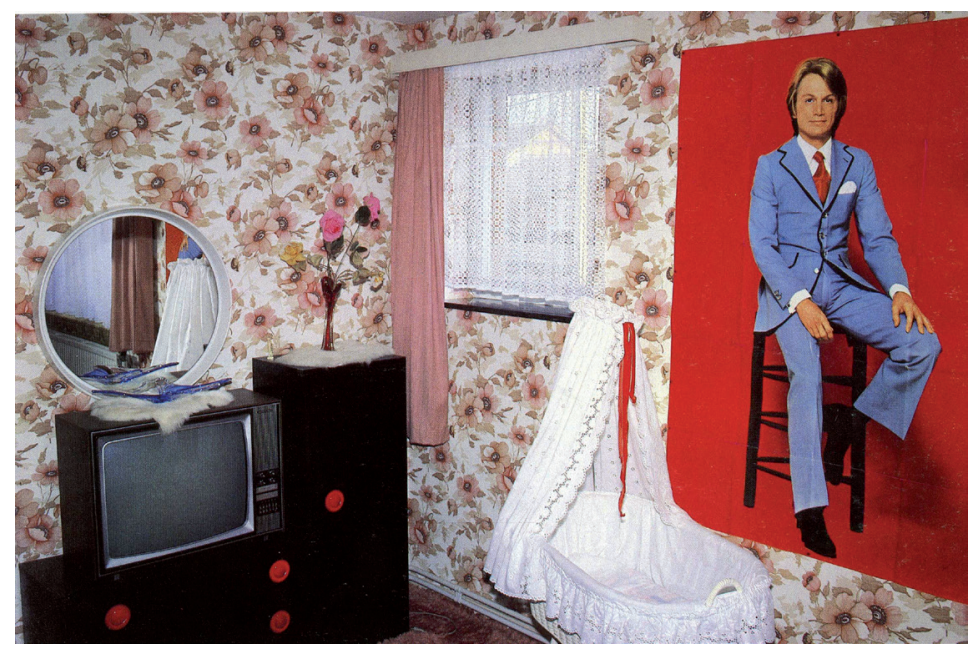

Figure 2. Intérieurs (1981 n.p.).

(C) François Hers.

De fait, les logements représentés sont bien tenus et témoignent d'une satisfaction relative des besoins quotidiens. Les éléments d'ameublement ne portent pas traces d'usure ou de détérioration. Ce que les photographies signalent en revanche, c'est que les classes populaires achètent des produits fabriqués par l'industrie pour la grande consommation - les mêmes choix, les mêmes modes d'organisation se trouvant pour ainsi dire répétés d'une image à l'autre. Les vues apportent somme toute un 
diagnostic de société, en révélant l'aliénation des classes sociales peu favorisées, telles qu'elles se manifestent au travers de leurs goûts. En sus de la surcharge de couleurs et de motifs, le cadrage serré des photographies contribue à créer un sentiment de relatif étouffement. Les images de François Hers, se rapprochant de constats cliniques, témoignent d'une volonté de ne pas enjoliver les choses, loin de toute forme d'empathie, afin de privilégier une approche politique.

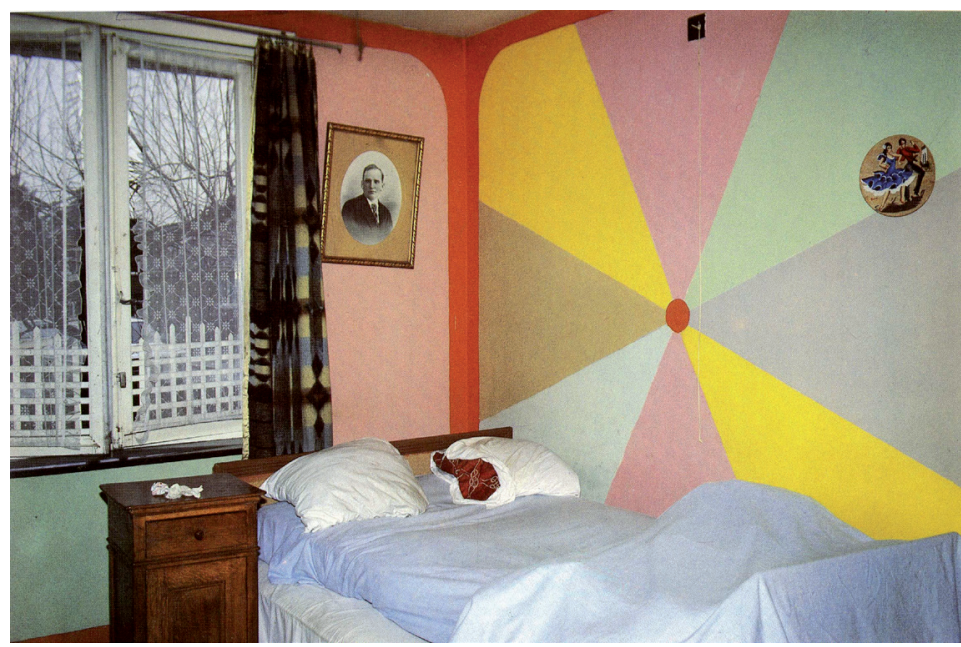

Figure 3. Intérieurs (1981 n.p.).

(C) François Hers.

Dans le même temps, les grands tirages qu'il expose au Centre Pompidou en 1981 monumentalisent les intérieurs. Les vues structurées en aplats colorés possèdent une richesse formelle: leur manque de profondeur, leur planéité, l'entrechoquement des motifs imprimés des tissus et des papiers peints peuvent faire lointainement penser aux toiles de Vuillard ou encore à celles de Matisse. Difficilement affiliable à un mouvement spécifique, François Hers initie donc, dès le début des années 80 , une posture hybride et féconde, entre art et sciences sociales. Ses images révèlent des options d'ameublement, liées à des manières de vivre qui relèvent d'une appartenance de classe. 


\section{Intérieurs, 1991}

Si Jean-Marc Tingaud appartient à la même génération que François Hers, son itinéraire est tout autre. Autodidacte, originaire du Morvan, il a beaucoup voyagé, même si une bonne part de son travail est ancrée dans un terroir et tournée vers la mémoire ${ }^{4}$. Les vues d'intérieurs populaires rassemblées dans l'ouvrage qu'il publie en 1991 aux éditions Contrejour ont été réalisées à Paris, Naples, Zagreb ou Portland... Ce sont des plans serrés sur des coins de fenêtre, des fragments de mur ou de plafond peuplés d'objets variés qui se présentent comme autant de miettes de vie accumulées. Les vues minutieusement réalisées à la chambre livrent toute une stratification d'objets quotidiens ou de reliques intimes. Elles se caractérisent par une rigoureuse fidélité chromatique, un savant respect des contrastes qu'autorise la technique de tirage du «dye transfer». ${ }^{5}$

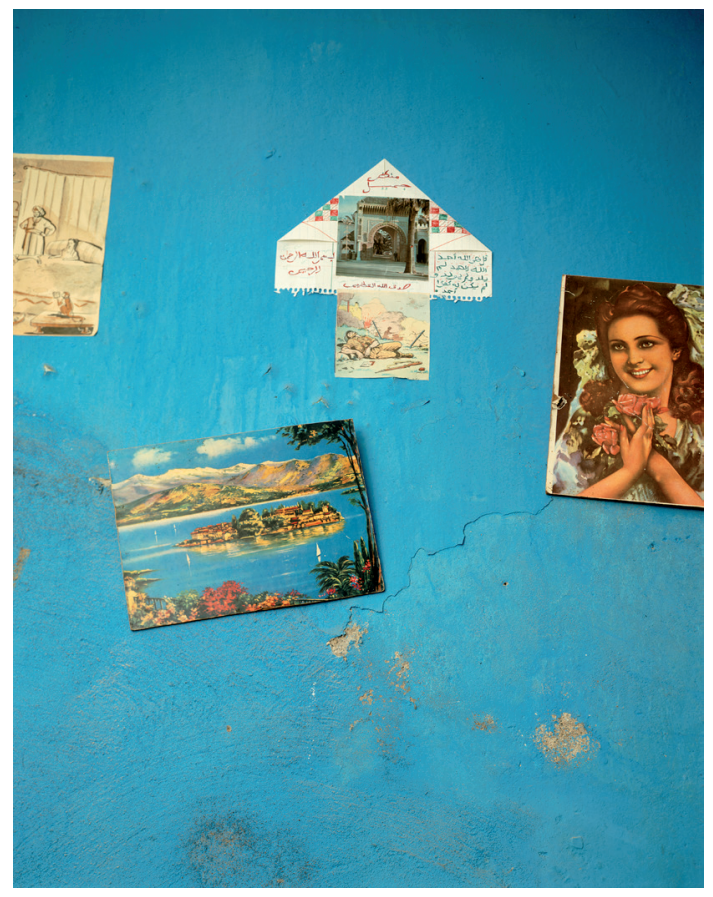

Figure 4. Intérieurs (1991 n.p.).

(C) Jean-Marc Tingaud.
Au côté d'objets utilitaires tels que balais, téléphones ou postes de radio, figurent des cartes postales, des portraits photographiques et des bibelots liés au souvenir. À chaque relique, des récits individuels paraissent potentiellement attachés. Pour les habitants de ces logements, bon nombre d'objets sont sans doute supports de mémoire, convoquant le souvenir, comme étaient susceptibles de le faire certains lieux matériels pour les orateurs de l'antiquité (Yates). La persistance des choses autour des personnes incarne pour ainsi dire leur capacité à rester les mêmes malgré la fuite du temps. La sociologue Véronique Dassié remarque notamment que «[q]uand le parcours biographique prend une nouvelle orientation, les objets prennent sens dans leur aptitude à matérialiser les satisfactions et les heurts des bifurcations d'une destinée»

${ }^{4}$ Ce tropisme est perceptible dans Gens du Morvan en 1978, Mémoires en 1986 ou Intérieurs en 1991.

${ }^{5}$ Procédé de tirage en couleur à partir d'une diapositive, qui permet au photographe d'ajuster séparément chaque couleur en vue d'un contrôle très précis du résultat final. 
(Dassié 342); ils prennent une importance particulière quand les vies ont été chahutées.

Les vues aux tonalités subtiles de JeanMarc Tingaud sont de format portrait - ce qui ne peut que favoriser le rapprochement des espaces considérés avec une (ou des) présence(s) humaine(s) évoquée(s) en creux. Leur composition, extrêmement concertée, ne se trouve pas centrée sur telle ou telle chose. Les photographies sectionnent au contraire certains objets pour mettre en évidence des espacements ou des proximités entre les choses. Elles rendent ainsi palpable l'organisation des objets et des reliques au sein du logis. Les décadrages et les décentrements, qui n'ont rien d'aléatoire, désignent des voisinages et isolent des fragments de syntagmes dans leurs articulations précises. L'attention se porte ainsi sur les jointures - les respirations d'une syntaxe vernaculaire.

Par leur composition calculée et leur traitement chromatique respectueux des nuances, les photographies de Jean-Marc Tingaud signent l'importance d'une «culture matérielle» telle que définie par les fondateurs de l'anthropologie. La

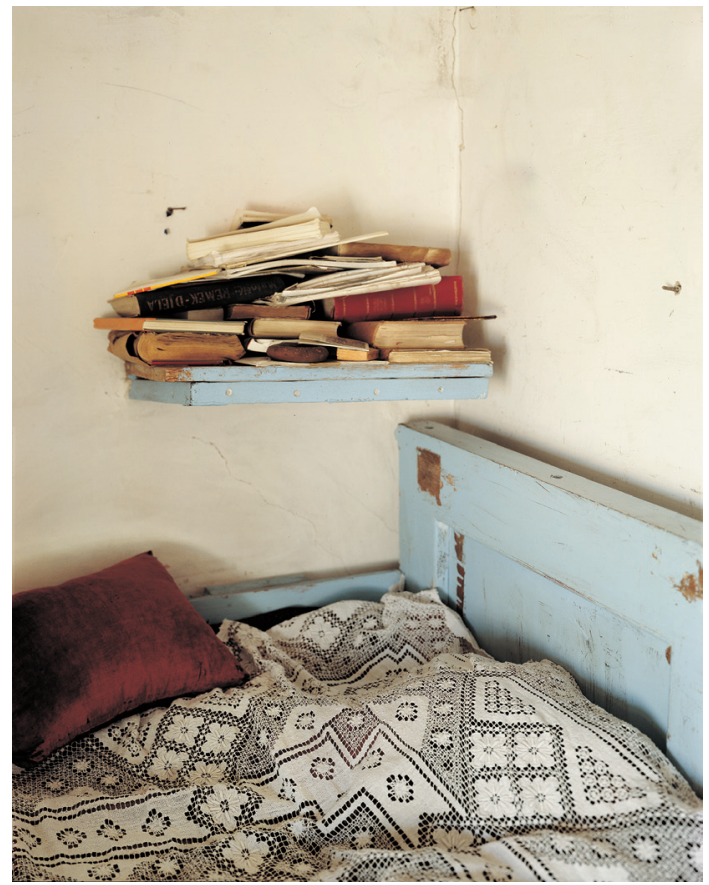

Figure 5. Intérieurs (1991 n.p.).

(C) Jean-Marc Tingaud. valeur des objets dans leur relation avec les corps a été soulignée par Marcel Mauss (1926), leur prise en compte donnant accès à une compréhension des cultures et des sociétés. Pour l'archéologue, l'ethnologue ou l'anthropologue, les objets sont des sources directes d'interprétation et de compréhension: selon un fonctionnement proche de la synecdoque, ils permettent de remonter vers des modes de vie. Utilitaires, les artefacts matériels sont habités de scenarii d'usage; plus gratuits, ils reflètent des trajectoires individuelles, des origines ou des compositions familiales. Les objets qui émaillent les photographies de Jean-Marc Tingaud sont de peu de valeur financière, mais ils paraissent gorgés d'affects. L'enchaînement des images au fil des pages de l'ouvrage donne à voir tout un bric-à-brac d'objets accumulés, offerts à une ethnographie du «minuscule» (Dassié). Il n'est pas indifférent que les images de Jean-Marc Tingaud soient réalisées en des aires géographiques très variées : c'est à chaque fois une culture, des traditions, des goûts différents qui se trouvent conviés. 


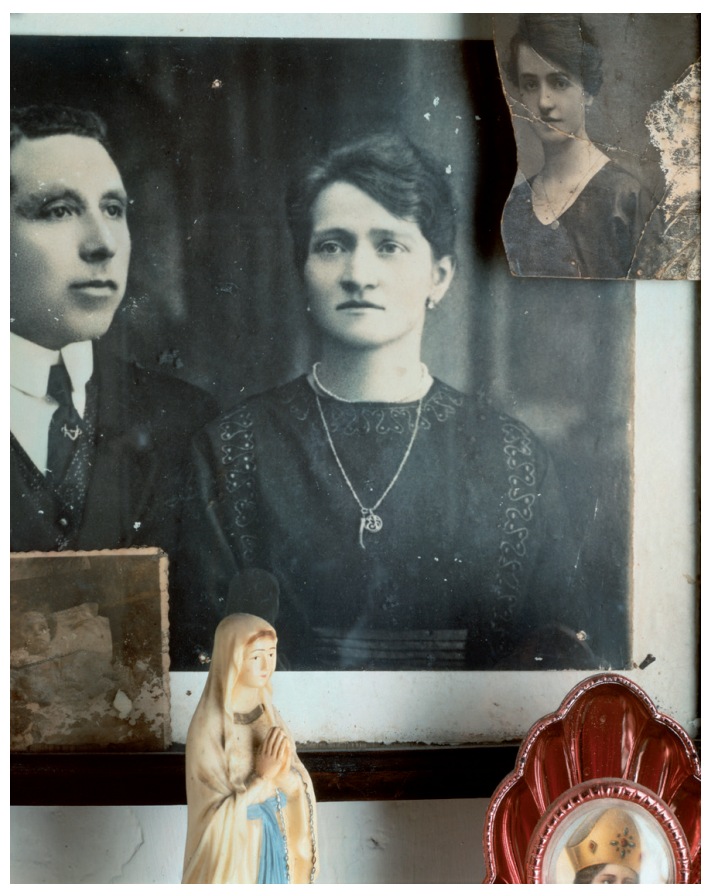

Figure 6. Intérieurs (1991 n.p.).

(C) Jean-Marc Tingaud.

Les choix opérés en matière de cadrage inclinent le spectateur à porter son attention sur des modalités d'association idiosyncrasique: les agencements particuliers que retiennent les vues de Jean-Marc Tingaud traduisent des manières singulières de faire avec les choses - qui relèvent du vernaculaire. En effet, les sujets s'approprient des héritages culturels en même temps qu'ils produisent des cultures particulières. Si les objets ont été fabriqués par d'autres, les syntaxes qui les organisent dans les intérieurs découlent de pratiques créatives de la vie ordinaire. Ces agencements sont le fruit de lents ajustements, de choix restreints et d'habitudes consenties. Ils témoignent de façons personnelles de «braconner» avec les objets, de «tactiques» banales mais inventives pour reprendre les termes utilisés par Michel de Certeau (1980). Les habitants opèrent de fait des détournements, des articulations, des espacements; ils organisent des échos dans des espaces localisés et personnels.

Les objets portent, pour certains, des traces d'usure; les murs sont craquelés, les photographies jaunies, les livres abîmés... Les choses révèlent les marques d'un corps à corps prolongé avec leurs propriétaires; de la sorte, elles paraissent presque faire partie intégrante des êtres qui les possèdent ; absents des photographies, ces derniers semblent néanmoins très présents en leur sein, tant les choses s'avèrent empreintes d'usages et d'affects matérialisés. Les images de Jean-Marc Tingaud laissent supposer combien les objets - et ce qu'ils portent en eux - participent d'une construction du sujet. Organisés en phrases, ces derniers se donnent comme matrices de subjectivation.

Davantage que dans les photographies de François Hers, l'accent se trouve ici porté sur l'intime et l'affectif. Le format portrait et la douceur des coloris s'y montrent propices, tout comme le cadrage sensiblement plus serré. Pourtant, la capacité à organiser les choses à sa façon dans un espace privé revient d'une image à l'autre, d'un pays à l'autre, signant somme toute une aptitude partagée des êtres humains à investir l'espace du logement en y articulant des objets selon des grammaires vernaculaires, 
des bricolages syntaxiques singuliers. L'individuel s'articule ainsi au collectif, au sein d'un travail dont le lyrisme n'est pas absent (Vadé 11-37).

\section{Intérieurs, 2011}

Plus récente, l'activité photographique d'Hortense Soichet porte de façon privilégiée sur les modes d'habitation, sa pratique artistique confinant à la sociologie ou à l'anthropologie; le plus souvent, elle combine prises de vue et entretiens libres, dont elle extrait des témoignages qui se trouvent ensuite présentés au côté de ses images; si la jeune femme expose, le livre se présente néanmoins comme son support de prédilection (Soichet 2015). Entre 2009 et 2010, elle réalise un travail d'enquête au sujet des logements de la Zone Urbaine Sensible de la Goutte d'Or à Paris - qui a été exposé sous diverses formes, et a abouti à l'ouvrage intitulé Intérieurs aux éditions Créaphis en 2011. Si d'autres travaux à certains égards comparables ont été réalisés depuis par Hortense Soichet, il sera principalement ici question de ce premier ouvrage.

Revendiquant 1'héritage d'Eugène Atget ${ }^{6}$ et de François Hers, Hortense Soichet s'attache prioritairement à l'appréhension d'une réalité sociale, celle du logement dans une Zone Urbaine Sensible ${ }^{7}$ qui n'est pas dans une situation périphérique, mais localisée dans un quartier de Paris bien desservi par les transports en commun. Le secteur est en mutation, les classes sociales les moins favorisées laissant peu à peu la place à des personnes au revenu plus confortable (Soichet 2015, 114). Il s'agissait pour la photographe de rendre compte de cette diversité des modes d'habiter, quand la gentrification des espaces publics du quartier n'était pas vraiment encore évidente (Fijalkow) et le changement plutôt discernable au sein des intérieurs.

En 1910, ce dernier réalise une série de photographies d'intérieurs parisiens.

Une zone urbaine sensible est un territoire infra-urbain défini en France par les pouvoirs publics pour être la cible prioritaire de la politique de la ville entre 1996 et 2014. 


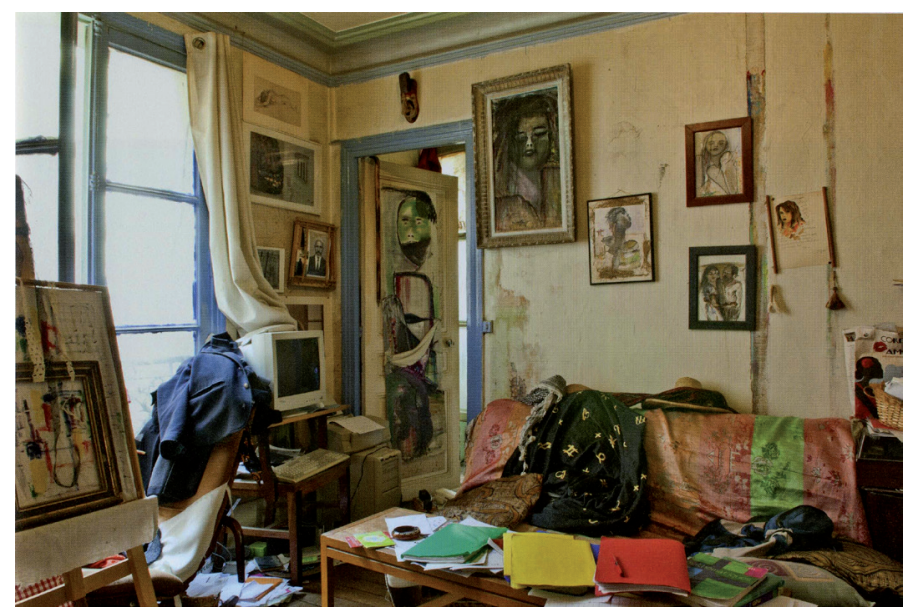

Figure 7. Intérieurs. Logements à la Goutte d'Or. «Rue de Suez, 1 habitant, 2 pièces, 42 m², 1994 » (2011 47).

(c) Hortense Soichet.

Pour Hortense Soichet, la première difficulté est d'entrer en contact avec les habitants. C'est grâce au bouche-à-oreille, par le biais d'associations ou encore par le truchement de la presse locale qu'elle tâche de parvenir à un corpus représentatif. Chaque visite obéit à un même protocole: les habitants sont interrogés, plus ou moins longuement, sur leur relation au quartier et à leur logement. Une photographie est faite (avec le plus de recul possible) de l'espace à vivre principal; puis plusieurs images dont le cadrage est plus serré sont prises des autres pièces (ou de zones restreintes de la même pièce quand le logement se réduit à cela). Le processus suppose la mise en confiance et la collaboration des habitants, les photographies réalisées étant systématiquement transmises aux occupants des lieux, qui peuvent éventuellement suggérer certaines modifications.

La suite des images en couleur et des textes inclus dans l'ouvrage se présente donc comme le fruit d'un patient travail de terrain. Les photographies de la pièce principale, de «format paysage» et cernées de blanc, sont proposées en belle page, tandis qu'en face figurent des informations factuelles - rue où se situe le logement, nombre d'habitants, nombre de pièces, superficie, date d'emménagement - ainsi qu'une ou deux phrases sélectionnées par Hortense Soichet au sein des propos recueillis sur place. Sur la double-page suivante, six photographies de format carré et de plus petite taille viennent épeler des détails du logement. Cette disposition restitue la durée d'une investigation des lieux. 


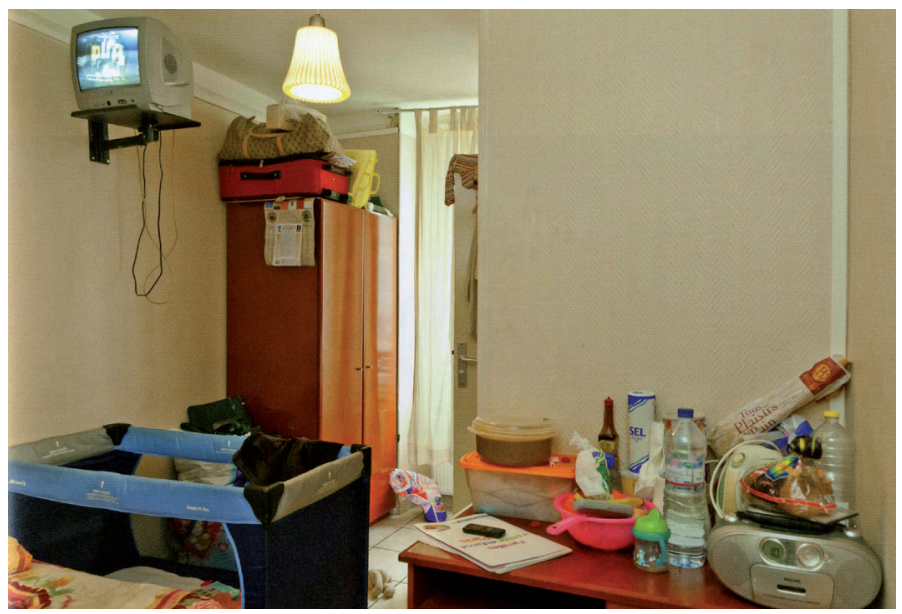

Figure 8. Intérieurs. Logements à la Goutte d'Or. «Rue Saint-Mathieu, 1 habitant, 1 pièce, 8 m², 2004 » (2011 163).

(C) Hortense Soichet.

Les vues de la pièce principale sont faites sous un éclairage naturel, au grand angle mais sans déformation, selon un point de vue diagonal le plus souvent, axial parfois. Elles sont nettes et leurs tonalités claires se montrent favorables à une relative mise en valeur du logement - qui se présente avant tout comme un espace de vie. Les habitants sont absents, mais les intérieurs représentés renvoient à des gestes vécus, des habitudes quotidiennes, des goûts et des activités. Parfois les fauteuils livrent l'empreinte des corps qui s'y sont assis; des tasses ou des cahiers ouverts renvoient à des actions entamées; chaque objet, si rudimentaire soit-il, semble avoir été choisi en fonction d'envies ou de besoins précis (Moles Théorie des objets, 33) et les agencements résulter d'une succession d'ajustements étroitement déterminés par les manières de vivre. Ces intérieurs se donnent comme les enveloppes de corps dont la présence est presque palpable, dans leur absence même. La relation aux choses repose en effet sur un accommodement routinier, de sorte qu'elles se trouvent pour ainsi dire "incorporées » par les êtres (Mauss 1934, 383-86) et occupent une place fondamentale dans la vie quotidienne. Leur agencement restitue ce qui est de l'ordre du faire, les corps se trouvant pour ainsi dire élargis aux objets, selon des conduites motrices, des contraintes d'accessibilité et des routines établies. Les vues d'Hortense Soichet suggèrent cette connexion physique des êtres à des supports matériels dans l'espace. 
À l'instar d'un certain nombre d'artistes contemporains (Caillet), Hortense Soichet emprunte une part de ses méthodes aux sciences humaines et sociales: sa démarche relève de l'enquête. À la manière d'un chercheur, elle sonde les façons diversifiées d'habiter la Goutte d'Or. Son travail se situe ainsi à la croisée de l'art et de la sociologie (ou de l'anthropologie), bousculant les cloisonnements établis et participant au renouvellement des méthodes utilisées en sciences humaines et sociales où l'approche globale se trouve délaissée au profit du parcellaire et de l'individuel (Méaux). Le regard permet de mesurer et de comprendre combien habiter, c'est investir de soi dans un espace qui devient abri personnel.

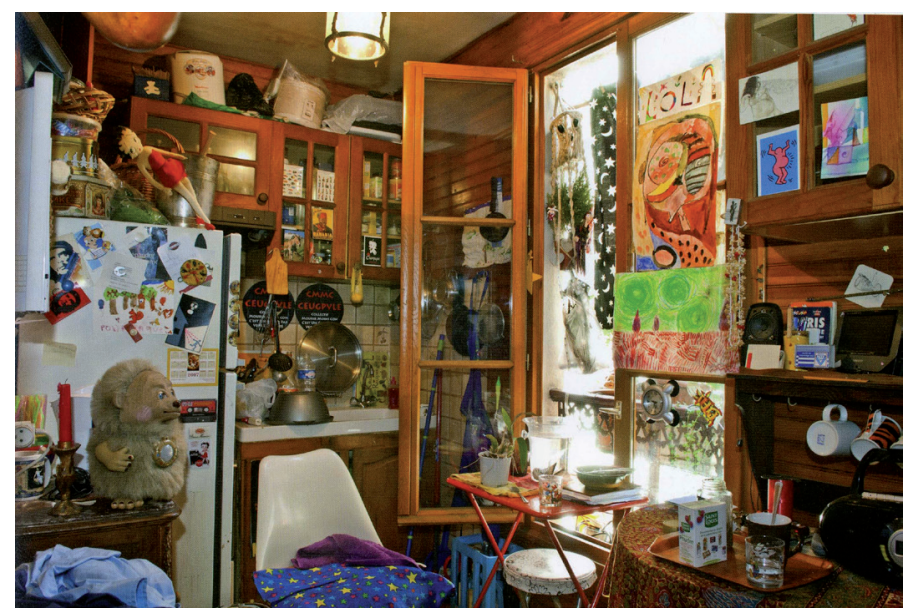

Figure 9. Intérieurs. Logements à la Goutte d'Or. «Rue Doudeauville, 2 habitants, 3 pièces, $65 \mathrm{~m}^{2}$, 2002» (2011 83). (c) Hortense Soichet.

L'ouvrage conjuguant informations verbales, paroles d'habitants et photographies de différents formats permet au lecteur de suivre le cheminement de l'enquête. C'est avec curiosité qu'il pénètre les lieux, qu'il compare les habitats, des plus cossus au plus déshérités, des plus rudimentaires aux plus sophistiqués. Il se surprend à être absorbé dans l'exploration de ces idiosyncrasies matérielles. Grâce à la confrontation des photographies et des propos rapportés, le spectateur a le sentiment de pouvoir se projeter dans l'action d'habiter ces logements. Le dispositif livresque l'amène à se couler dans des aménagements vernaculaires, des logements spacieux ou exigus, désordonnés ou bien rangés, un peu comme y invitent les magazines de décoration illustrés de clichés qui donnent envie de vivre dans les 
pièces qu'ils figurent, sauf que les intérieurs photographiés par Hortense Soichet se présentent bien loin du luxe et des dernières tendances du design ou du high-tech.

Un tel dispositif travaille à rendre sensible la démarche poursuivie par la photographe et à soumettre à l'examen un positionnement qui enjambe les champs de l'art et de la sociologie (ou de l'anthropologie). Il ouvre l'œuvre à une réception consciente des procédures adoptées - dont l'influence du mouvement conceptuel n'est pas absente. Il participe aussi à une forme d'ouverture démocratique du processus de l'enquête - que John Dewey appelait de ses vœux (Dewey). En effet, les spectateurs se trouvent conviés à participer d'une volonté de comprendre les manières d'habiter.

\section{Syntaxes domestiques}

Échelonnées sur quatre décennies et renvoyant à différents moments de l'histoire récente du médium, de ses évolutions techniques en matière de traitement des couleurs et de ses usages artistiques, ces trois séries se font écho: la photographie s'y présente en effet, à chaque fois, comme une modalité privilégiée d'approche de la culture matérielle, donnant à voir des intérieurs populaires vides de leurs occupants, mais dont les organisations singulières permettent d'inférer des modes de vie. Quand le travail de François Hers tend à proposer le constat assez cru d'un aménagement des logements déterminé par une appartenance de classe, les photographies de Jean-Marc Tingaud, plus esthétisantes voire lyriques, signent le rapport de la stratification des objets accumulés à la mémoire. Quant au travail plus récent d'Hortense Soichet, il relève explicitement de l'enquête. Néanmoins, dans les trois séries, les espaces domestiques se trouvent livrés à une auscultation minutieuse. Les intérieurs sont enregistrés par la photographie qui rabat l'organisation des objets dans l'espace tridimensionnel à la surface de la représentation, faisant ainsi ressortir l'accumulation des artefacts et leurs dispositions singulières dans le champ. Manifestement, pour ces trois photographes, les modalités d'agencement des logements s'avèrent dignes d'être explorées dans toute leur complexité, des pratiques ordinaires se trouvant ainsi reconsidérées.

Chacune à leur manière, les trois séries travaillent à documenter la culture matérielle domestique de milieux relativement populaires. Les artefacts figurant dans les intérieurs mis en image sont généralement de peu de valeur; de fabrication industrielle, ils ressortissent souvent au kitsch; ils traduisent des niveaux de vie variés, mais rarement très aisés. Les objets accumulés semblent répondre à des besoins quotidiens et sont en rapport avec des usages concrets, les acquisitions étant déterminées par le coût et l'accessibilité des produits. Les agencements sont également pour partie dictés par des patrons comportementaux. Selon des degrés divers, François Hers, Jean-Marc Tingaud et Hortense 
Soichet rendent sensibles ces déterminations; les séries photographiques font ressortir que les manières d'habiter se trouvent conditionnées par des modes, des contraintes économiques et des normes sociales (Certeau Xxxv).

Mais, dans le même temps, comme l'écrit Michel de Certeau, «les usagers "bricolent” avec et dans l'économie culturelle dominante les innombrables et infinitésimales métamorphoses de sa loi en celle de leurs intérêts et de leurs règles propres» (xxxix): au travers de «tactiques» diversifiées, ils composent des syntaxes singulières afin de «faire avec» les espaces et les objets. Les trois séries photographiques ici prises en considération font ressortir que les combinatoires complexes réalisées par les occupants des lieux transcrivent, au travers des objets, des parcours biographiques singuliers. Elles mettent aussi en évidence combien, à partir d'acquisitions obéissant à des déterminations économiques et sociales, les habitants développent des modalités actives d'appropriation et d'organisation des choses qui témoignent d'une réelle inventivité.

Au sein des images, les aménagements intérieurs se présentent dans un inachèvement permanent: intimement liés à des manières de faire privées, ils manifestent des formes continues d'adaptation aux besoins, de bricolage à des fins utilitaires ou d'ingéniosité décorative - auxquelles les vues rendent diversement hommage. Mais, par leurs différences d'approche mêmes, les séries photographiques de François Hers, Jean-Marc Tingaud et Hortense Soichet conduisent également à penser, de façon dialogique et articulée, toute la complexité du «vernaculaire», tel qu'il se trouve pris entre kitsch (lié à l'emploi d'objets découlant d'une production de masse) et vitalité d'une inventivité ordinaire. 


\section{Ouvrages CITÉS}

ADORNO, Theodor. Théorie esthétique. 1969. Trad. de l'allemand par Marc Jimenez. Paris: Klincksieck, 2011.

ATGET, Eugène. Intérieurs parisiens. Début du XXe siècle: artistiques, pittoresques et bourgeois. Paris: Musée Carnavalet, 1982.

BESSE, Jean-Marc. Habiter. Un monde à mon image. Paris : Flammarion, 2013.

BOLTANSKI, Luc. Énigmes et complots. Une enquête à propos d'enquêtes. Paris: Gallimard (NRF), 2012.

BONHOMME, Max. «L'œuvre photographique de François Hers. En quête d'un "art de la démocratie"». Paris: Mémoire de l'École du Louvre présenté sous la direction de Dominique de Font-Réaulx et de Michel Poivert, 2014.

CAILLET, Aline. L'Art de l'enquête. Savoirs pratiques et sciences sociales. Paris: Mimésis (Art, esthétique, philosophie), 2019.

CERTEAU, Michel de. L'Invention du quotidien 1. Arts de faire. 1980. Paris : Gallimard (Folio), 1990.

DASSIÉ, Véronique. Objets d'affection. Une ethnologie de l'intime. Paris : CTHS (Le regard de l'ethnologue), 2010.

DEWEY, John. Logic: The Theory of Inquiry. New York: Henry Holt and Company, 1938.

FIJALKOW, Yankel. «Rêveries sociologiques dans les appartements de ma Goutte-d'Or». In Hortense Soichet. Intérieurs. Logements à la Goutte d'Or. Paris: Créaphis, 2011. 171-173.

FOSTER, Hal. Le Retour du réel. Situation actuelle de l'avant-garde. 1996. Trad. de l'anglais par Yves Cantaine, Frank Pierobon et Daniel Vander Gucht. Bruxelles: La Lettre volée, 2005.

HERS, François. Intérieurs (en collaboration avec Sophie Ristelhueber). Bruxelles: Éditions des Archives d'Architecture Moderne, 1981.

HERS, François. «La tête dans la mort». Libération 153 (10 novembre 1981): 13.

HERS, François. «Avec ceux de la P. J. ». Photo 173 (1982): 31-35.

ILLICH, Ivan. Dans le miroir du passé. Paris : Descartes \& Cie, 1994.

KALIFA, Dominique, dir. Romantisme 149 («Enquête et culture de l'enquête au XIX'e siècle», 2010).

LEFEBVRE, Henri. Critique de la vie quotidienne 1. Introduction. 1947. Paris: L'Arche, 1977.

LUSSAULT, Michel. L'Homme spatial. La construction sociale de l'espace humain. Paris: Seuil (La couleur des idées), 2007. 
MARESCA, Sylvain. La Photographie, un miroir des sciences sociales. Paris: L'Harmattan, 1996.

MAUSS, Marcel. Manuel d'ethnographie. 1926. Paris : Petite bibliothèque Payot, 2002.

MAUSS, Marcel. «Les techniques du corps». 1934. Sociologie et anthropologie. Paris: PUL, 1968.

MÉAUX, Danièle. Enquêtes. Nouvelles formes de photographie documentaire. Trézélan: Filigranes éditions, 2019.

MOLES, Abraham. Psychologie de l'espace (en collaboration avec Élisabeth Rohmer). Paris : Casterman, 1972.

MOLES, Abraham. Théorie des objets. Paris : Éditions universitaires, 1972.

SCHOONBRODT, René. «Images du logement social». In François Hers. Intérieurs (en collaboration avec Sophie Ristelhueber). Bruxelles : Éditions des Archives d'Architecture Moderne, 1981. 20-25.

SOICHET, Hortense. Intérieurs. Logements à la Goutte d'Or. Paris: Créaphis, 2011.

SOICHET, Hortense. «Les territoires de l'ordinaire: photographies de logements et paroles d'habitants». Revues des Sciences Humaines 319 («Espaces phototextuels» dir. Danièle Méaux, 2015) : 113-125.

TINGAUD, Jean-Marc. Intérieurs. Paris : Contrejour, 1991.

VADÉ, Yves. «L'émergence du sujet lyrique à l'époque du romantisme». Figures du sujet lyrique. Dir. Dominique Rabaté. Paris : PUF (Perspectives littéraires), 1996. 11-37.

YATES Frances. L'Art de la mémoire. 1966. Paris: Gallimard (NRF), 1987. 